\title{
Spontaneous Resorption of a Large Lumbar Disc Herniation within 4 Months
}

\section{TO THE EDITOR}

A 48-year-old man presented in March 2013 with a 20-day history of low back and right leg pain with no obvious cause. There was no history of surgical trauma. Neurological examination showed no deficits. A straight leg-raising test was positive at $30^{\circ}$ on the right side. The Japanese Orthopaedic Association (JOA) score for low back pain was 4 points (1). Magnetic resonance imaging (MRI) of the lumbar spine suggested the pres- ence of a large intervertebral disc herniation at L4/L5, a typical site for this abnormality (Fig. 1). Surgery to remove the herniated disc was recommended, but the patient declined. Consequently, the patient was treated conservatively, which included bed rest, with steroidal anti-inflammatory drugs for 2 months and oral administration of Chinese medicine for 4 months. In July 2013, he was re-examined and had no complaints, with a JOA score of 28 points. A second MRI study showed com-

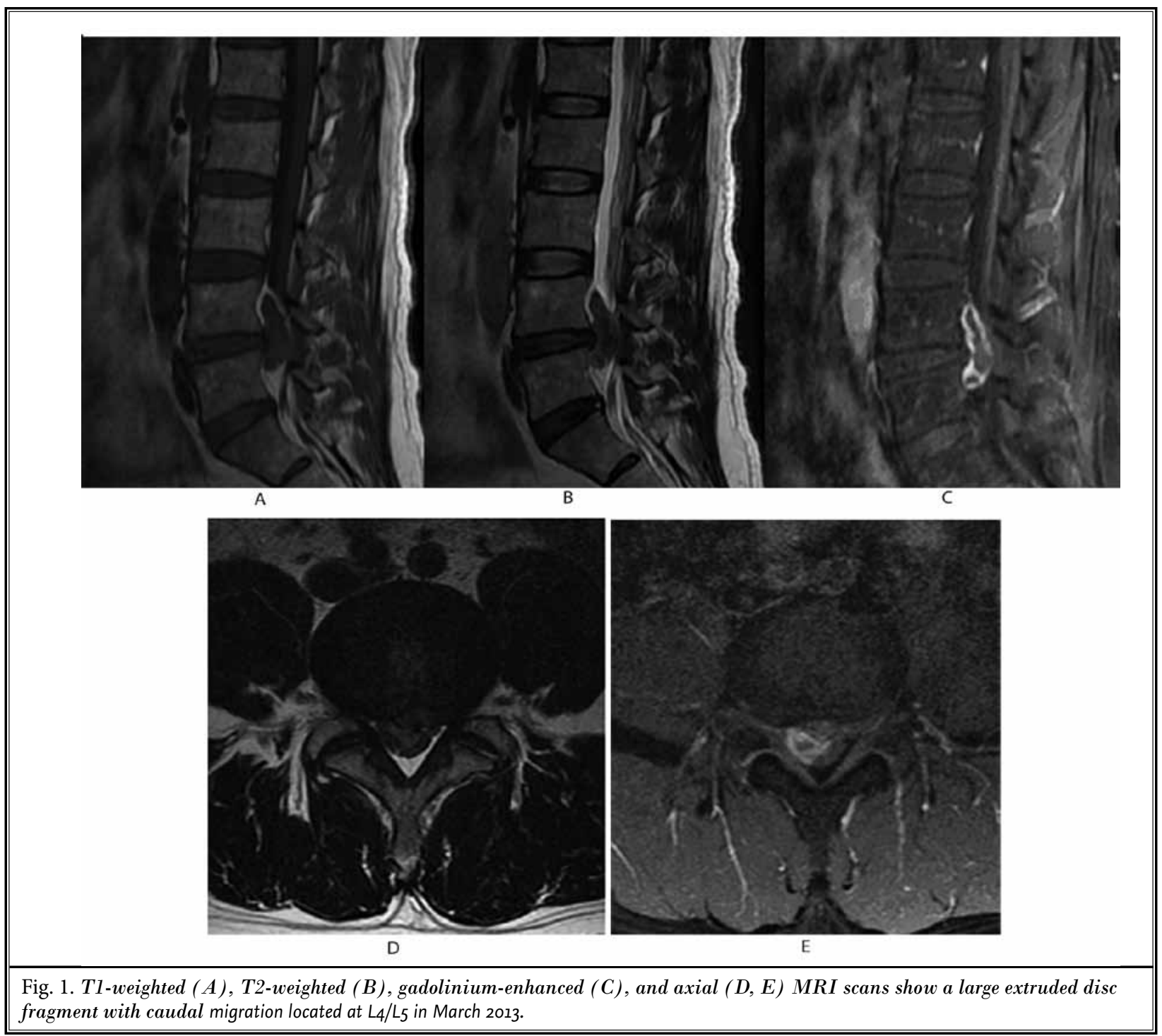


plete disappearance of the extruded fragment that had been located at L4/L5 (Fig. 2).

\section{Conclusions}

Key, in 1945, was the first to report spontaneous resorption of extruded herniated disc material. His report was followed by others that demonstrated this phenomenon at various regions of the spine (2). Recent advances in MRI technology have facilitated follow-up of intervertebral disc herniation regarding spontaneous resorption and its correlation with alleviation of neurological symptoms (3). Most patients suffering from radiculopathy caused by intervertebral disc herniation heal spontaneously, without surgical intervention. Since Guinto et al (4) first presented a case of spontane- ous resorption of a lumbar herniated disc demonstrated by computed tomography (CT) in 1984, an increasing number of similar cases have been described $(5,6)$. The spontaneous disappearance of intervertebral disc herniation is well documented, yet the exact mechanism of this process remains unresolved.

Komori et al (7) reported that the more the herniated nucleus pulposus migrated, the greater was the subsequent decrease in size. Henmi et al (8) noted that large protruded disc fragments diminished more than small fragments. They thought it may be due to the larger disc fragments having more water content, especially in patients younger than 40 years of age. Several studies have reported the ratio of spontaneous resorption of herniated discs. Recently, a large study was re-

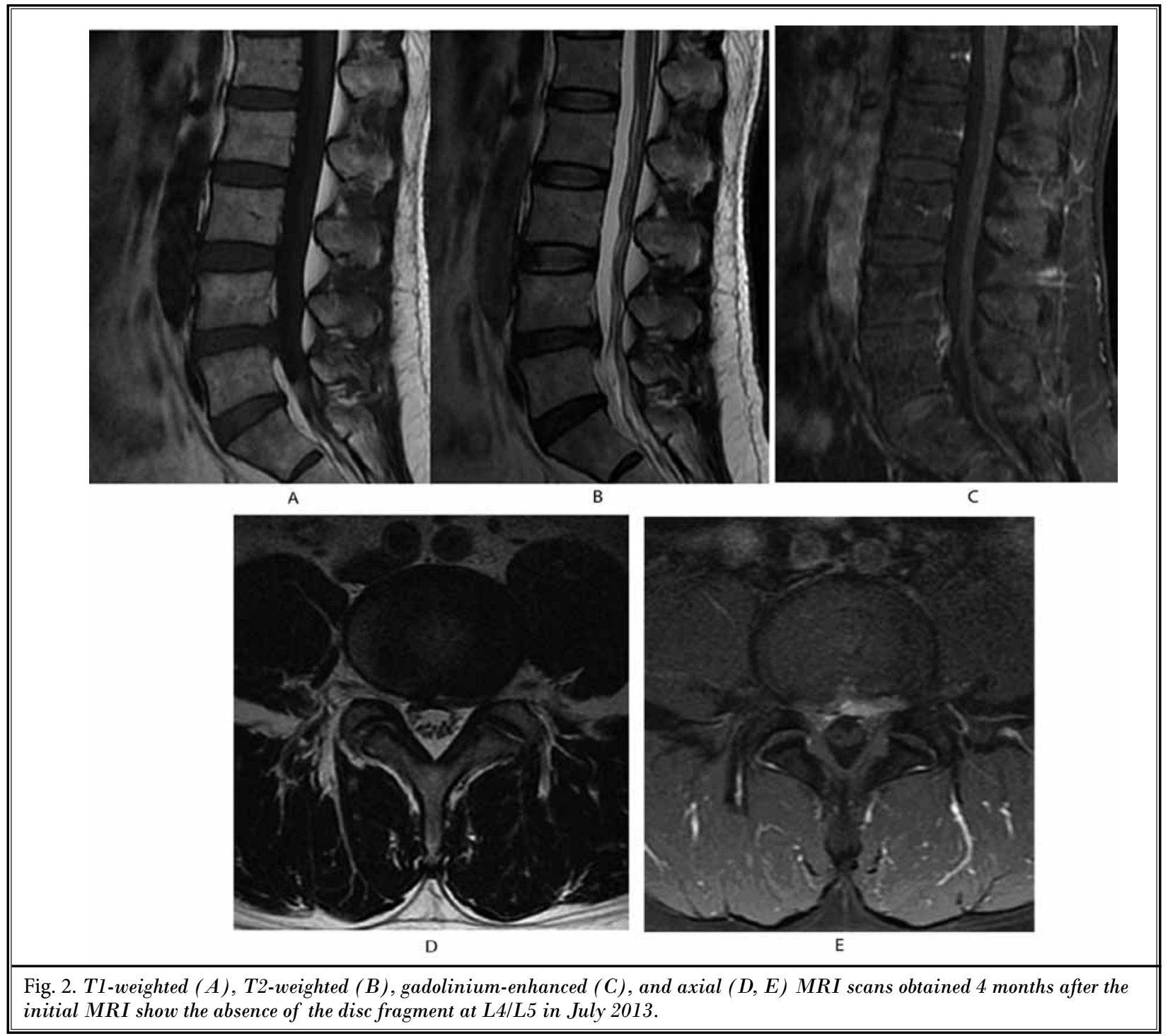


ported by Autio et al (9) in which 68 of 160 enrolled patients $(42.5 \%)$ documented by lumbar MRI had a diminished volume of herniated lumbar discs 2 months after their occurrence. In other studies, the occurrence of spontaneous resorption of herniated lumbar discs was around $35 \%-63 \%$, on average, over a period of 6 months to one year $(7,9)$.

The differential diagnosis of these epidural lesions includes cysts, abscesses, tumors, and hematomas. Cysts demonstrate a low-intensity signal on T1-weighted MRI and a very high-intensity signal on T2-weighted MRI. Patients with abscesses and infection have a fever, and clinical laboratory test results show abnormal values indicating inflammatory reactions. Tumors exhibit various intensities on T1- and T2-weighted MRI (10). However, tumors typically do not show improvement during their natural course. Komori et al (11) found that a herniated mass that exhibited rim enhancement on gadolinium-enhanced MRI scans disappeared or markedly decreased in $75 \%-100 \%$ of cases. Autio et al (9), in fact, suggested performing gadolinium-enhanced MRI for these patients to predict the possibility of spontaneous regression of their herniated discs.

Various theories exist to explain the mechanism behind spontaneous resorption of these disc fragments. Three popular mechanisms have been described in the literature. The first mechanism involves dehydration and shrinkage of the herniated nucleus pulposus (12). The second mechanism proposes that the herniated disc may retract back into the intervertebral disc space. Theoretically, retraction occurs when the herniated disc protrudes through the annulus fibrosus without separating from it. The third mechanism proposes that enzymatic degradation and phagocytosis of the sequestrated disc material occurs as the result of an inflammatory reaction and neovascularization (13). Once the nucleus pulposus sequestrates into the epidural vascular space of the spine, it is recognized as a foreign body by the autoimmune system and an inflammatory reaction is induced that leads to neovascularization, enzymatic degradation, and macrophage phagocytosis (13). Subsequently, production of matrix proteinases and increased cytokine levels play a role in the spontaneous regression process (14).

Although orthopedic surgeons and neurosurgeons are gradually recognizing the phenomenon of spontaneous regression of disc herniation, it is still a relatively unknown phenomenon to internists and general surgeons who sometimes encounter patients with back pain. Our case shows that large lumbar herniated discs can indeed be resorbed spontaneously. Conservative treatment should be considered when cauda equina syndrome and progressive motor weakness are absent during the acute stage of a lumbar herniated disc. The treatment protocols and options have been gradually changing. For such patients, we suggest that treatment be conservative during the initial course of the disease. Surgical intervention should be limited to those who still have moderate to severe neurological deficits or intractable low back and leg pain after 6 weeks of conservative treatment (15).

Jin-Tao Liu, MD

Department of Orthopaedic Surgery

Suzhou Hospital of Traditional Chinese Medicine

Jiangsu, China

Longhua Hospital

Shanghai University of Traditional Chinese Medicine Shanghai, China

Xiao-Feng Li, MD

Longhua Hospital

Shanghai University of Traditional Chinese Medicine Shanghai, China

Peng-Fei Yu, MD

Department of Orthopaedic Surgery

Suzhou Hospital of Traditional Chinese Medicine Jiangsu, China

Xiao-Chun Li, MD

Department of Orthopaedic Surgery

Suzhou Hospital of Traditional Chinese Medicine Jiangsu, China

Qiang Qian, MD

Department of Orthopaedic Surger

Suzhou Hospital of Traditional Chinese Medicine Jiangsu, China

Guan-Hong Liu, MD

Department of Orthopaedic Surger

Suzhou Hospital of Traditional Chinese Medicine Jiangsu, China

Zhen-Han Yu, MD

Department of Orthopaedic Surgery

Suzhou Hospital of Traditional Chinese Medicine Jiangsu, China 
Qi-Han Ma, MD

Department of Orthopaedic Surgery

Suzhou Hospital of Traditional Chinese Medicine Jiangsu, China

\author{
De-Zhi Tang, MD \\ Longhua Hospital \\ Shanghai University of Traditional Chinese Medicine \\ Shanghai, China \\ E-mail: dztang702@126.com
}

\author{
Hong Jiang, MD \\ Department of Orthopaedic Surgery \\ Suzhou Hospital of Traditional Chinese Medicine, \\ 889 Wuzhongxi Road \\ Suzhou, Jiangsu 215009, China. \\ E-mail: doctorhong@yeah.net
}

\section{References}

1. Toyone T, Takahashi K, Kitahara H, Yamagata M, Murakami $\mathrm{M}$, Moriya $\mathrm{H}$. Visualisation of symptomatic nerve roots. Prospective study of contrast-enhanced MRI in patients with lumbar disc herniation. The Journal of Bone and Joint Surgery British Volume 1993; 75:529-533.

2. Key JA. Intervertebral disk lesions are the most common cause of low back pain with or without sciatica. Annals of Surgery 1945; 121:534.

3. Keskil S, Ayberk G, Evliyaoglu C, Kizartici T, Yucel E, Anbarci $\mathrm{H}$. Spontaneous resolution of "protruded" lumbar discs. MIN 2004; 47:226-229.

4. Guinto FC, Jr., Hashim H, Stumer M. CT demonstration of disk regression after conservative therapy. AJNR 1984; 5:632-633.

5. Autio RA, Karppinen J, Kurunlahti M, Haapea M, Vanharanta $\mathrm{H}$, Tervonen $\mathrm{O}$. Effect of periradicular methylprednisolone on spontaneous resorption of intervertebral disc herniations. Spine 2004; 29:1601-1607.

6. Matsumoto M, Chiba K, Ishikawa M, Maruiwa H, Fujimura Y, Toyama Y. Relationships between outcomes of conservative treatment and magnetic resonance imaging findings in patients with mild cervical myelopathy caused by soft disc herniations. Spine 2001; 26:1592-1598.

7. Komori H, Shinomiya K, Nakai O, Yamaura I, Takeda S, Furuya K. The natural history of herniated nucleus pulposus with radiculopathy. Spine 1996; 21:225-229.

8. Henmi T, Sairyo K, Nakano S, Kanematsu Y, Kajikawa T, Katoh S, Goel VK. Natural history of extruded lumbar intervertebral disc herniation. JMI 2002; 49:40-43.

9. Autio RA, Karppinen J, Niinimaki J, Ojala R, Kurunlahti

M, Haapea M, Vanharanta H, Tervonen O. Determinants of spontaneous resorption of intervertebral disc herniations. Spine 2006; 31:1247-1252.

10. Tarukado K, Ikuta K, Fukutoku Y, Tono O, Doi T. Spontaneous regression of posterior epidural migrated lumbar disc fragments: case series. The Spine Journal 2013. [Epub ahead of print]

11. Komori H, Okawa A, Haro H, Muneta T, Yamamoto H, Shinomiya K. Contrast-enhanced magnetic resonance imaging in conservative management of lumbar disc herniation. Spine 1998; 23:67-73.

12. Slavin KV, Raja A, Thornton J, Wagner FC, Jr. Spontaneous regression of a large lumbar disc herniation: Report of an illustrative case. Surgical Neurology 2001; 56:333-336; discussion 337.

13. Geiss A, Larsson K, Rydevik B, Takahashi I, Olmarker K. Autoimmune properties of nucleus pulposus: An experimental study in pigs. Spine 2007; 32:168-173.

14. Tsarouhas A, Soufla G, Katonis P, Pasku D, Vakis A, Spandidos DA. Transcript levels of major MMPs and ADAMTS-4 in relation to the clinicopathological profile of patients with lumbar disc herniation. European Spine Journal 2011; 20:781-790.

15. Weinstein JN, Tosteson TD, Lurie JD, Tosteson AN, Hanscom B, Skinner JS, Abdu WA, Hilibrand AS, Boden SD, Deyo RA. Surgical vs nonoperative treatment for lumbar disk herniation: The Spine Patient Outcomes Research Trial (SPORT): A randomized trial. JAMA 2006; 296:24412450. 